\title{
Power control for multipoint cooperative communication with high-to-low sinr scenario
}

\author{
Hui Zhang ${ }^{1,2^{*}}$, Ying Fang ${ }^{2}$, Yifeng $X_{i e}{ }^{2}$, Hong $W^{2}$ and Yanyan Guo ${ }^{3}$
}

\begin{abstract}
In order to solve the power control problem for multipoint cooperative communication with high-to-low SINR scenario, the cooperative SINR receiving model is established. Moreover, considering its non-convex property, a novel power control algorithm is proposed, which is based on the geometric programming and a series of convex approximations are taken to achieve the global optimization in high-to-low SINR scenario. The numerical results show the power of cellular users can be brought into a global optimization range whether users in high-SINR area or low-SINR area compared with the existing algorithm, also the CDF of users' SINR is optimized, and its SINR coverage distribution could be balanced in varying degrees, which improve the fairness and mitigate the inter-cell interference effectively.
\end{abstract}

Keywords: CoMP, OFDMA, Inter-cell interference, Power control, Cooperative communication

\section{Introduction}

Recently, convex optimization theory gradually become a hot tool to solve the hard problems in communication area, for many such problems can be converted into the form of convex optimization. In brief, convex optimization mainly refers to the minimization of a convex objective function subject to convex constraints, and some modern software's have been developed into solve convex problems, such as CVX, SeDuMi, YALMIP, et al. [1]. However, the rate maximization problem is not yet amendable to a convex formulation for the interference channel in actual systems [2]. Especially, due to the frequency reuse in cellular networks and the limitation of signal to interference plus noise ratio (SINR), it's a long standing open problem in interference-limted wireless networks to achieve weighted throughput maximization through power control $[2,3]$. According to this open problem, some related research work have been done in single antenna scenario. For example, Chiang et al. [4] gives a single-antenna-based power control algorithm by means of geometric programming (GP) method, which divides the scenarios into two aspects, directly takes GP in high-

\footnotetext{
* Correspondence: zhangh.nku@gmail.com

${ }^{1}$ State Key Laboratory of Networking and Switching Technology (Beijing

University of Posts and Telecommunications), Beijing, China

${ }^{2}$ Wireless Communication Lab, College of Information Technical Science,

Nankai University, 94 Weijin Road, Nankai District, Tianjin, China

Full list of author information is available at the end of the article
}

SINR region, while adopts a successive convex programming and equivalent signomial programming (SP) condensation algorithm to solve it in low-SINR region. Otherwise, Qian et al. [3] proposes a MAPEL (Multiplicative linear fractional programming-based power allocation) algorithm to achieve global optimality for a non-convex wireless single-antenna-based power control problem. The computation times of MAPEL algorithm drastically increase when accuracy increases, and MAPEL algorithm needs more convergence time with high-complexity.

With the development of novel technologies, there are some new challenges for the traditional power control method. On the one hand, it's still difficult to the straightforward application of single antenna power control in the multiple-input multiple-output (MIMO) context due to coordination between receiving antennas and nonlinear dependence between interference and eigen-spaces of the channel matrices. Considering this situation, Chen et al. [5] proposes an iterative channel inversion power control algorithm for the uplink of cellular MIMO spatial multiplexing systems, but not consider the power control problem with cellular cooperation scenario. On the other hand, recently cellular cooperative communication for multiple base stations and multiple users is drawing attention as a solution to achieve high system throughput in cell-edge for the 
future mobile communication systems, such as coordinated multipoint (CoMP) transmission, cooperative beam, cooperative resource control, cooperative transmission, cooperative relaying, etc [6-8]. As discussed in the IMT-advanced standardizing groups, it is expected to be essential for cooperative communication technologies in the next generation cellular networks [7]. With this background, Fodor et al. [9] gives a near optimum power control method under fairness constraints in CoMP transmission systems based on Lagrangian penalty function, which aims to improve the transmitting power allocation efficiency in multicell spatial multiplexing wireless systems. Considering the objective function is non-convex for the PHY constraints, [9] takes use of the link capacity approximation and replaces it into the objective function, which is cited from the rate approximation inequality in $[10,11]$. By means of this approximation of PHY constraints, [9] changes this nonconvex power control problem into a convex optimization problem to solve. However, the rate approximation condition needs the high SINR situation, and such approximation is hard to application in the low SINR condition. Moreover, it actually gives an analysis of single-point transmission scenario still without giving a theoretical derivation of CoMP scenario.

In 2005, Chiang [12] gives an explanation about why is GP useful for general communication systems, respectively from stochastic models and deterministic models. Also, Chiang et al. [4] gives an introduction that GP can be used to efficiently compute the globally optimal power control in many of these problems. As a special case, GP is also suitable for cooperative communication systems. Specifically, considering the characteristic of power control problem in CoMP systems, it needs to optimize the objective function with some other data stream from different base stations/users (such as throughput, power strength, SINR, etc.). The GP method could play an effective role in these optimization problems. For GP, its product operation can be easily converted to the summation operations of logarithm, written as log-sum-exp function. Based on it, the nonconvex problem can be converted into a convex optimization problem. Moreover, its Lagrange duality gap is zero under mild conditions and the global optimum can always be computed very efficiently [13].

Considering this situation, by means of GP, we propose an optimized power control algorithm with high-to-low SINR scenario for cooperative communication under MIMO cellular system. Our contributions include the following aspects: Compared with [5,9], the cooperative SINR receiving model is established and the cooperative base station sets are analyzed in our method, then the algorithm is extended into cooperative communication. In order to solve the non-convex property, the proposed algorithm is based on the GP method and a series of convex approximations are taken to achieve the global optimization in highto-low SINR scenario, which makes our method could be applied in both high SINR and low SINR scenario. The numerical results show the power of cellular users can be brought into a global optimization range whether users in high-SINR area or low-SINR area compared with the existing algorithm, also the CDF of users' SINR is optimized, and its SINR coverage distribution could be balanced in varying degrees, which improve the fairness and mitigate the inter-cell interference effectively.

The rest of this paper is organized as follows: The system model is introduced in System model. The overview of power control problem in MIMO system is made in Power control problem. The power control is analyzed and solved by GP respectively in Power control analysis by GP. Then a series of convex approximations is described in A series of convex approximation. The performance analysis is given in Performance analysis. Finally, the conclusion is made in Conclusion.

\section{System model}

As shown in Figure 1, it gives the network topology of cellular cooperative communication system, where several access points (APs) are connected into eNodeBs and some cell-edge users are served by the cooperative AP.

\section{Link Model}

Define $x_{k} \in \mathbb{C}^{N_{t} \times 1}$ is the data vector that is also assumed to be zeromean, normalized and uncorrelated, $E\left(x_{k} x_{k}^{\dagger}\right)=\mathbf{I}_{N_{t}}$. In the uplink, $\beta_{k, i}$ denotes a scalar coefficient depending on the total transmit power $P_{k}$ of user $k$, written as

$$
\beta_{k, i}=\sqrt{\frac{P_{k} d_{k, i}^{-\rho} X_{k, i}}{N_{t}}}
$$

where $X_{k, i}$ is the lognormal shadow fading and $d_{k, i}$ is the distance between the $k^{\text {th }}$ user and the $i^{\text {th }}$ base station. $H_{\mathrm{ki}}$ denotes the $\left(N_{r}, N_{t}\right)$ channel transfer matrix, $T_{k, i}$ denotes the MS- $k\left(N_{t}, N_{t}\right)$ diagonal power loading matrix. In order to keep the total transmit power as a constant, $T_{k, i}$ should satisfy

$$
E\left(T_{k, i}^{+} T_{k, i}\right)=\sum_{i=l}^{N_{t}}\left|T_{k, i}^{(i, j)}\right|^{2}=N_{t}
$$

Besides, assume $n_{k}$ is an $N_{r} \times 1$ additive with Gaussian noise vector at the $k^{\text {th }}$ base station with zero mean and the covariance matrix $R_{n_{k}}$ is defined as (3).

$$
R_{n_{k}}=E\left(n_{k} n_{k}^{\dagger}\right)=\sigma_{n}^{2} I_{N_{r}}
$$




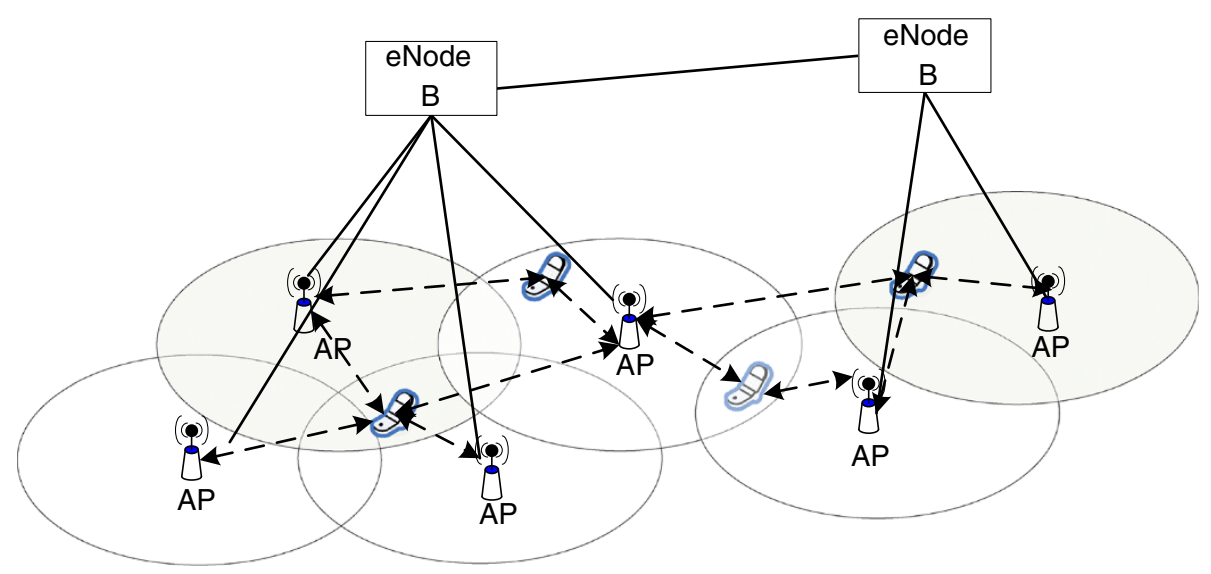

Figure 1 Network topology of cellular cooperative communication system.

Further, we define the coordinated transmission point (CTP) sets $\xi$, constructed by some cooperative APs. For cell $i$, if it is in the CTP sets, written as $i \in \xi$. Similarly, for cell $j$, if it isn't in the CTP sets, written as $j \in \bar{\xi}$. On this basis, the received signal $Y_{k}$ at the $k^{\text {th }}$ AP is represented as

$$
Y_{k}=\sum_{i \in \xi} \beta_{k, i} H_{k, i} T_{k, i} x_{k, i}+\sum_{j \in \bar{\xi}} \beta_{k, j} H_{k, j} T_{k, j} x_{k, j}+n_{k}
$$

Particularly, for single cellular signal without considering the cooperative transmission scenario, the received signal $y_{k}$ can be simplified as (5). Moreover, the dimension of $y_{k}$ and $y_{k}$ is according to the sent signal (original signal), which is respectively an expression of the received signal, whether it's in the form of the vector or not depends on the sent signal.

$$
y_{k}=\beta_{k, k} H_{k, k} T_{k, k} x_{k, k}+\sum_{j \neq k} \beta_{k, j} H_{k, j} T_{k, j} x_{k, j}+n_{k}
$$

\section{Linear MMSE Receiver}

Considering the linear MMSE receiver, assume $G_{k}$ denotes an MMSE weighting matrix, the received signal $\mathrm{y}_{k}$ is estimated as $\hat{x}_{k}$, that is

$$
\hat{x}_{k}=\mathbf{G}_{k} y_{k}
$$

The MMSE weighting matrix $G_{k}$ is equivalent to (7) with equal power allocation case. Specially, $\mathrm{T}_{\mathrm{k}}=\mathrm{I}_{N_{\mathrm{t}}}$.

$$
\begin{aligned}
G_{k}= & \frac{1}{\beta_{k, k}} T_{k, k}^{\dagger} H_{k, k}^{\dagger}\left(H_{k, k} T_{k, k} T_{k, k}^{\dagger} H_{k, k}^{+}\right. \\
& \left.+\sum_{j \neq k} \frac{\beta_{k, j}^{2}}{\beta_{k, k}^{2}} H_{k, j} T_{k, j} T_{k, j}^{\dagger} H_{k, j}^{\dagger}+\frac{\sigma_{n}^{2}}{\sigma_{k, k}^{2}} I_{N_{r}}\right)^{-1} \\
= & \frac{1}{\beta_{k, k}} H_{k, k}^{\dagger}\left(H_{k, k} H_{k, k}^{\dagger}+\right) \sum_{j \neq k} \frac{\beta_{k, j}^{2}}{\beta_{k, k}^{2}} H_{k, j} H_{k, j}^{\dagger}+\frac{\sigma_{n}^{2}}{\sigma_{k, k}^{2}} I_{N_{r}}
\end{aligned}
$$

On the other hand, we denote $\epsilon_{\text {MMSE }}$ as the MMSE estimation error matrix $\left(N_{r} \times N_{r}\right)$ for the $k$-th base station, that is $[5,9]$

$$
\epsilon_{M M S E}=\left\{\left(\mathbf{I}+\mathbf{T}_{k}^{\dagger} \mathbf{R}_{H_{k}} \mathbf{T}_{k}^{\dagger}\right)^{-1}\right\}_{(s, s)}
$$

Based on the MMSE estimation error matrix $\epsilon_{M M S E}$, the post-processing SINR by the linear MMSE receiver is denoted as $[14,15]$ :

$$
\hat{\gamma}_{k}=\frac{1}{\epsilon_{M M S E}}-1
$$

Furthermore, we assume the noise power is irrelevant with the number of cells. Considering the spatial diversity gain from each cooperative cell set, the joint postprocessing SINR could be derivated from Eq.(9), which is defined as

$$
\gamma_{k}=\sum_{i \in \xi} \frac{1-\epsilon_{M M S E}}{\epsilon_{M M S E}}
$$

\section{Power control problem}

Nowadays, it's a common problem for power control in MIMO systems, which may include many different actual scenarios. For example, non-cooperative power control [16], power control subjected to objective function [17], power control in cooperative communications [18], and so on. In this section, the non-cooperative power control and the cooperative power control subjected to objective function are respectively introduced as two major problems.

\section{Non-Cooperative Power Control in MIMO systems}

In MIMO system, the power allocation for user- $k$ in multi-cellular system should satisfy following inequality 
based on [5], which is under the equal allocation assumption.

$$
\begin{aligned}
P_{k} \geq & \frac{d_{k, k}^{\rho} \Gamma_{k}}{X_{k, k}}\left(\sum_{j \neq k} P_{j} d_{k, j}^{-\rho} X_{k, j} \mu_{\max }\left(\boldsymbol{\Omega}_{k, j, 1}\right)\right. \\
& \left.+N_{t} \sigma_{n}^{2} \mu_{\max }\left(\boldsymbol{\Omega}_{k, j, 2}\right)\right)
\end{aligned}
$$

where $\Gamma_{k}$ denotes as a given SINR target value for user- $k$. Moreover, we define $\boldsymbol{\Lambda}=\operatorname{dig}\left\{\Gamma_{1}, \Gamma_{2}, \cdots, \Gamma_{k}\right\}$, and $\mu_{\max }(\cdot)$ is the maximum eigenvalue of a Hermitian matrix. Besides, $\boldsymbol{\Omega}_{k, j, 1}, \boldsymbol{\Omega}_{k, j, 2}$ is respectively defined as

$$
\begin{aligned}
& \boldsymbol{\Omega}_{k, j, 1}=\left(\mathbf{H}_{k, k}^{\dagger} \mathbf{H}_{k, k}\right)^{-1} \mathbf{H}_{k, k}^{\dagger} \mathbf{H}_{k, j} \mathbf{H}_{k, j}^{\dagger} \mathbf{H}_{k, k}\left(\mathbf{H}_{k, k}^{+} \mathbf{H}_{k, k}\right)^{-1} \\
& \boldsymbol{\Omega}_{k, j, 2}=\left(\mathbf{H}_{k, k}^{+} \mathbf{H}_{k, k}\right)^{-1}
\end{aligned}
$$

According to [5], in a cellular MIMO system with $\mathrm{K}$ cells, the post-processing SINR for the $k^{\text {th }}$ user with linear MMSE receiver can be defined as follows:

$$
\begin{aligned}
\gamma_{k, \min } & =\operatorname{Min}_{s \in\left[1, N_{t}\right]} \gamma_{k, s} \\
& \geq \frac{P_{k} d_{k, k}^{-\rho} X_{k, k}}{\mu_{\max }\left(\sum_{j \neq k} P_{j} d_{k, j}^{-\rho} X_{k, j} \boldsymbol{\Omega}_{k, j, 1}+N_{t} \sigma_{n}^{2} \boldsymbol{\Omega}_{k, j, 2}\right)}
\end{aligned}
$$

On this basis, the optimized power factor for power control problem in MIMO systems can be derived as $[5,19]$

$$
\mathbf{P}^{*}=(\mathbf{I}-\mathbf{\Lambda} \mathbf{F})^{-1} \mathbf{\Lambda} \mathbf{N}
$$

where $\mathrm{N}$ is a $N_{t} \times 1$ dimension noise vector, and $\mathrm{F}$ is defined as

$$
\mathbf{F}_{k, j}=\left\{\begin{array}{c}
0, k=j \\
\left(\frac{\beta_{k, j}}{\beta_{k, k}}\right)^{2} \mu_{\max }\left(\boldsymbol{\Omega}_{k, j, 1}\right), k \neq j
\end{array}\right.
$$

\section{Cooperative Power Control in MIMO System subjected to Objective Function}

In cooperative communication area, the objective of power minimum and rate maximum is contradictory in power control problem. In order to mitigate the uplink inter-cell co-frequency interference, it's necessary to reduce the power in transmitter, making the power minimum ahead of actual service. Otherwise, in order to improve transmission rate, it's necessary to raise the sum of rate in each sub-link. It's a non-convex for such problem, and nowadays many research work aims to solve its global optimization value [3]. Considering the gap between capacity and actual transmission rate, by means of an SINR-gap approximation in the Shannon's theory, the transmission rate $r_{k}$ is written as [4]

$$
r_{k}(\mathbf{P})=W_{k} \log \left(1+K \gamma_{k}(\mathbf{P})\right)
$$

where $W_{k}$ is the bandwidth for user $k$, and also denotes user $k$ 's weight, the coefficient $K$ is the gap to capacity and always smaller than 1 . Specially, $K$ is equivalent to $-1.5 / \log (5 B E R)$ for M-QAM, with $B E R=10^{-3}$.

On this basis, the power allocation problem in cooperative communication generally aims to solve the weighted throughput extremum and mitigate the intercell interference, including two types: One needs to maximize the sum rates with constraint capacity, written as Type I. The other needs to minimize the sum power at the user side with constraint rate, written as Type II.

$$
\underset{P, \gamma}{\operatorname{Maximize}} \sum_{\mathrm{k}} r_{k}
$$

Type I : subject to $r_{k} \leq c_{k}(P)$

$$
\begin{array}{r}
\sum_{k} P_{k} \leq P_{\text {tot. }} \\
\underset{P, \gamma}{\text { Minimize }} \sum_{k} P_{k} \\
\text { subject to } r_{k}^{\text {target }} \leq r_{k} \\
\sum_{k} P_{k} \leq P_{\text {tot. }}
\end{array}
$$$$
\text { Type II : subject to } r_{k}^{\text {target }} \leq r_{k}
$$

Over the years, the weighted throughput extremum (WTE) is one of plagued problem in interference-limited wireless networks, for mutual interference in the transmission link constitutes a non-convex optimization problem [1]. The existing research focus on the high SINR scenario, in whose condition the WTE problem can be transmitted into convex optimization by mathematical approximation method. However, for the low SINR scenario, the constraint conditions for such approximation method is difficult to set up, making no sense for the existing solution schemes in general scenario. In order to solve the WTE problem with high-to-low SINR scenario, it's necessary to study the non-convex power control algorithm with global convergence.

\section{Power control analysis by geometric programming}

In MIMO system, both the capacity maximization problem subject to power constraint and the power minimization problem subject to SINR constraints can be formulated as a convex problem. However, due to the interference channel where multiple transmitters and multiple receivers interfere with each other in a shared medium, the rate maximization problem like (18) isn't yet amendable to a convex formulation [2]. Nowadays, GP is introduced into solve non-convex optimization problem, and the above WTE problem can also be efficiently sovled by GP ways. 
Generally, GP is a type of mathematical optimization problem characterized by objective and constraint functions that have a special form, which is also a class of nonlinear optimization and its standard form is still a non-convex optimization problem, because the polynomials are not convex functions [20,21]. However, by means of a logarithmic transition, the variables and multiplicative constraints can respectively be turned into logarithmic variables and plus constraints, which is a convex form for the log-sum-exp function is convex. On this basis, it enables to take convex optimization methods to solve the above GP problem although it's nolinear and non-convex in the form.

Considering the objective function $r_{k}=W_{k} \log \left(1+K \gamma_{k}\right)$ in (18) is non-convex, a GP-based power control algotithm is given in [12] to solve this WTE problem. In that paper, the transmitting rate is approximated by $r_{k}=W_{k}$ $\log \left(K \gamma_{k}\right)$ when the SINR is in the high regime that is much larger than $0 \mathrm{~dB}$ in each link, or the spreading gain in CDMA systems is large [12]. With this approximation, (18) is transformed into the convex optimization problem in the form of GP after log-sum-exp variables change [4]. Figure 2 shows the processing flow for the power control problem solved by existing GP method [22]. First, a threshold SINR value is defined for the power control problem, and then the scenario is devided into two parts, respectively high SINR scenario and low SINR scenario. As shown in Figure 2, the original power control problem is directly changed into a GP problem under high SINR scenario, but under low SINR scenario, it needs two steps of transition, first by the SP method and then through complementary condensed. After these prepare, it becomes a traditional GP problem. But how to set the high-to-low SINR threshold is not given in [22]. Due to the change speed of user location and channel variability, it's hard to define the SINR threshold for a uncertain randomness. Moreover, this method only gives a conceptual optimization strategy but in actual scenario it inevitably lead to a certain lag and the defined complexity of SINR threshold.
However, the high SINR scenario assumption isn't always valid in actual wireless networks, and it gives a segment solution by existing GP method, which is only a local optimization solution for the SP condensation [3]. Moreover, the algorithm complexity increases for the interior steps of SP and complementary GP. In this paper, we propose another transition way from the original non-convex power control problem into the GP problem, which can get a global optimization solution by means of inequality iterative approximation to the objective.

Specially, the processing flow for power control problem solved by proposed GP method is shown in the Figure 3, which includes three parts, respectively logexponential variable transformation, rate-SINR inequality approaching and GP problem. In log-exponential variable transformation, take any positive real number $a$ as an example, assume $\tilde{a}=\log a$, then the exponential variable transformation can be taken as $a=e^{\tilde{a}}$. In rateSINR inequality approaching, for example, we choose the inequality $\phi \log (x)+\varphi \leq \log (1+x)$, where $\phi$ and $\varphi$ belong to real restriction parameters. Then this inequality can be taken to approach the throughput by iteration, and the original problem can be changed into GP problem.

Due to such method does not depend on SINR threshold, it can be found that the proposed power control approach is not only specific to cooperative networks but also can be applied to more general scenarios. Under high-to-low SINR scenario, take a logexponential variable transformation for the critical parameters in power control problem, turning the original problem into a convex optimization problem that the log-sum-exp function belongs to convex case [23]. For the objective function, search a tight iterative inequality between transmission rate and SINR, making the formula related with SINR approach to the rate. Then, solve this GP problem to get the optimized power and SINR value. Section V describe the calculation process in detail.

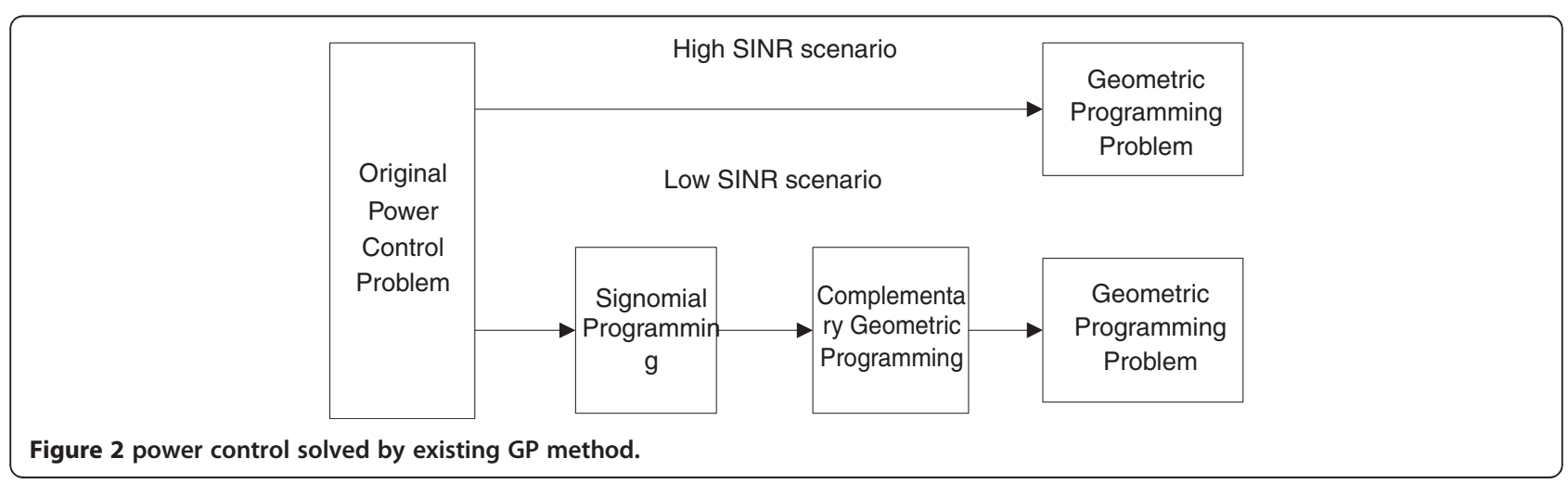




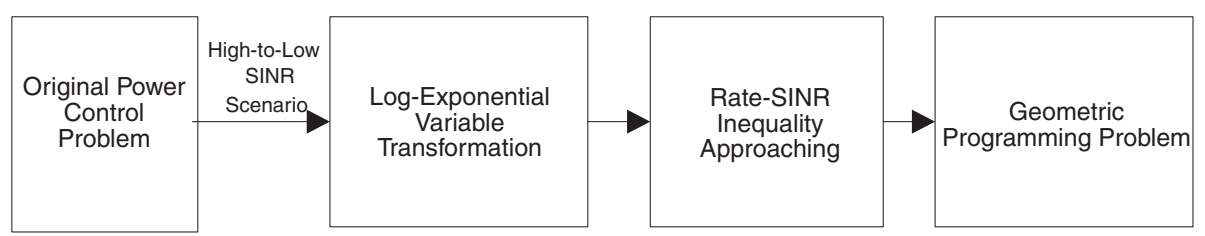

Figure 3 power control solved by proposed GP method.

\section{A series of convex approximation}

It is worth mentioning that an inequality related the rate in [2] is given to solve resource allocation under highSINR approximation. Specially, it is also taken in [10] to solve the rate approximation problem by means of Lagrangian penalty function method. For this algorithm, it is constrainted with high SINR situation for such mathimatical approximation and only has optimum solution under high SINR condition. Moreover, the algorithm complexity increases for computing the tighted coefficients. What's important, these coefficients aim to let the formula value related withlog $(S I N R)$ tightly approximate into $\log (1+S I N R)$. When directly taking an equivalent approximation from $\log (S I N R)$ to $S I N R$, the approaching condition would be changed, making the computation error under these related coefficients may be larger and not in the form of optimal approximated values, which is neglected in [9]. Considering this problem, a global optimization solution is proposed for global SINR approximation in this paper. By means of the inequality $\phi \log (x)+\varphi \leq \log (1+x)$, where $\phi$ and $\varphi$ are approximation constants at $x=x_{0}$, respectively

$$
\phi=\frac{x_{0}}{1+x_{0}}, \varphi=\log \left(1+x_{0}\right)-\phi \log \left(x_{0}\right)
$$

For $\phi_{k} \log (K \gamma)+\varphi_{k} \leq \log (1+K \gamma)$, where it should satisfy $\phi_{k}=\frac{K \gamma_{k}}{1+K \gamma_{k}}, \varphi_{k}=\log \left(1+K \gamma_{k}\right)-\phi_{k} \log \left(K \gamma_{k}\right)$, so we can get

$$
\tilde{R}_{k}\left(P ; \phi_{k}, \varphi_{k}\right) \triangleq \mathrm{W}\left(\phi_{\mathrm{k}} \log \left(\mathrm{K} \gamma_{\mathrm{k}}\right)+\varphi_{\mathrm{k}}\right)
$$

Based on (20), a convex approximation of objective function is presented to solve power control optimization problem with High-to-Low SINR Scenario, as shown in (21).

$$
\begin{aligned}
& \underset{P, \gamma}{\operatorname{Maximize}}-\mathrm{P}_{k} \\
& \text { subject to } r_{k}^{\text {target }} \leq \tilde{R}_{k}\left(P ; \phi_{k}, \varphi_{k}\right) \\
& \sum_{k} e^{\tilde{P}_{k}} \leq P_{\text {tot. }} .
\end{aligned}
$$

Considering the Lagrange dual function [24], we get

$$
\begin{aligned}
L\left(\gamma_{k}, \mathrm{P}_{k}\right)= & -\mathrm{P}_{k}+\mu_{k}\left[W\left(\phi_{k} \log \left(K \gamma_{k}\right)+\varphi_{k}\right)-r_{k}^{\text {target }}\right] \\
& +\lambda_{k}\left[P_{\text {tot. }}-\sum_{k} \mathrm{P}_{k}\right]
\end{aligned}
$$

where $\mu_{k}, \lambda_{k}$ are the Lagrangian multipliers. By means of Lagrange dual function, we can get the update form according to the Newton iterative method:

$$
\begin{aligned}
P_{k}^{n(s+1)}= & \frac{\mu_{k}^{(s)} \phi_{k} W}{K\left(1+\lambda_{k}^{(s)}\right)} \\
\mu_{k}^{(s+1)}= & {\left[\mu_{k}^{(s)}+\epsilon_{\mu}\left(r_{k}^{\text {target }}-\sum_{n} W\left(\phi_{k} \log \left(K \gamma_{k}^{(s)}\right)\right.\right.\right.} \\
& \left.\left.\left.+\varphi_{k}\right)\right)\right]^{+} \\
\lambda_{k}^{(s+1)}= & {\left[\lambda_{k}^{(s)}+\epsilon_{\lambda}\left(\sum_{n} P_{k}^{n(s)}-P_{\text {tot. }}\right)\right]^{+} }
\end{aligned}
$$

where $[\cdot]^{+}=\max (0,$.$) , and its proof is given in the$ Appendix. On the other hand, we still need to prove the formula (23) is convergent, which can be proved from three aspects, respectively positivity, monotonicity and scalability. The proof of its convergence is given as follows: Since $W / K$ is a constant value, we only need to make an analysis of $\mu_{k} \phi_{k} /\left(1+\lambda_{k}\right)$. Let $\mathbf{J}(P)=\mu_{k} \phi_{k} /\left(1+\lambda_{k}\right)$, where $\mu_{k}=\left[\mu_{k}+\epsilon_{\mu}\left(r_{k}^{\text {target }}-\sum_{n} W\left(\phi_{k} \log \left(K \tilde{\gamma}_{k}\right)+\varphi_{k}\right)\right)\right]^{+}$, and $\lambda_{k}=\left[\lambda_{k}+\epsilon_{\lambda}\left(\sum_{n} P-P_{t o t .}\right)\right]^{+}$, then we prove that (23) satisfies positivity, monotonicity and scalability.

Positivity: Each component in $\mathbf{J}(P)$ is non-negativity.

Monotonicity: Assume $\tau \geq 1$, let $Q=\tau P$, so $P \leq Q$, we have

$$
\begin{aligned}
\mathbf{J}(P) & =\frac{\mu_{k} \phi_{k}}{1+\left[\lambda_{k}+\epsilon_{\lambda}\left(\sum_{n} P-P_{\text {tot. }}\right)\right]^{+}} \\
& \geq \frac{\mu_{k} \phi_{k}}{1+\left[\lambda_{k}+\epsilon_{\lambda}\left(\sum_{n} \tau P-P_{\text {tot. }}\right)\right]^{+}}=\mathbf{J}(Q)
\end{aligned}
$$

Scalability: Also take $\tau \geq 1$, so we can get

$$
\begin{aligned}
\tau \mathbf{J}(P) & =\frac{\tau \mu_{k} \phi_{k}}{1+\left[\lambda_{k}+\epsilon_{\lambda}\left(\sum_{n} P-P_{\text {tot. }}\right)\right]^{+}} \\
& \geq \frac{\mu_{k} \phi_{k}}{1+\left[\lambda_{k}+\epsilon_{\lambda}\left(\sum_{n} \tau P-P_{\text {tot. }}\right)\right]^{+}}=\mathbf{J}(\tau P)
\end{aligned}
$$


Based on the above analysis, the proposed power control algorithm with high-to-low SINR scenario by GP method is shown as following:

\section{Algorithm 1: Power Control with High-to-Low SINR Scenario by GP Method}

1. Initialize for all users. When $t=0, \phi_{k}^{(0)}=K \gamma_{k}^{(0)}\left[1+\gamma_{k}^{(0)}\right]^{-1}$, $\varphi_{k}^{(0)}=\log \left[\left(1+K \gamma_{k}^{(0)}\right)\left(K \gamma_{k}^{(0)}\right)^{-\phi_{k}}\right]$

2. For $s=1: 1: N, \phi_{k}^{(s)}=K \gamma_{k}^{(s)}\left[1+\gamma_{k}^{(s)}\right]^{-1}$,

$$
\varphi_{k}^{(s)}=\log \left[\left(1+K \gamma_{k}^{(s)}\right)\left(K \gamma_{k}^{(s)}\right)^{-\phi_{k}}\right]
$$

3. Solve the subproblem (7) by Lagrange dual function $L\left(\gamma_{k}, P_{k}\right)$ to give solution

4. Compute $\gamma_{k}, P_{k}$ according to the following formula $P_{k}=e^{\tilde{P}_{k}}, r_{k}=e^{\tilde{r}_{k}}$

5. If $\frac{\left\|P_{k}^{(s)}-P_{k}^{(s-1)}\right\|}{P_{k}^{(s-1)}} \geq \eta$, then go to step 2, update $\phi_{k}^{(s)}, \varphi_{k}^{(s)}$ and repeat iteration. Else, stop iteration, output $\gamma_{k}, P_{k}$

\section{Performance analysis}

In simulation, we consider 19-cell system, the cell radius is $1 \mathrm{~km}$, while the ISD takes $500 \mathrm{~m}$. The channel model chooses rayleigh fading and the bandwidth takes $5 \mathrm{MHz}$. The thermal noise density is $-174 \mathrm{dBm} / \mathrm{Hz}$. The form of nTX_nRX antennas is 2_2. Moreover, the macroscopic pathloss is $128.1+37.6 \log 10(\mathrm{R})$. The scheduler scheme takes round robin [25]. Moreover, the CVX tool is also taken in the analysis of GP problem [23]. The performance analysis of the proposed algorithm is given as follows:

Figure 4 shows the individual power levels in seven cases for Fodor algorithm and the proposed algorithm, where each case represents a single independent Monte Carlo simulation with the same parameter definition. The algorithm level-1 denotes the users in cell-center that decrease power to reach the target rate. In the initial state, the cellcenter power is distributed from $70 \mathrm{~mW}$ to $100 \mathrm{~mW}$. After taking such two algorithms respectively, the cell-center power for each user is distributed from $30 \mathrm{~mW}$ to $50 \mathrm{~mW}$. Moreover, compared with Ford algorithm, the effectiveness of inhibiting cell-center power is relatively weak by the proposed algorithm, which enables to raise the actual SINR values for cell-center users. On the other hand, the algorithm level-2 denotes the users in cell-edge that increase power to reach the target rate. In the initial state, the celledge power is distributed from $20 \mathrm{~mW}$ to $40 \mathrm{~mW}$. After taking such two algorithms respectively, the cell-edge power for each user is distributed from $60 \mathrm{~mW}$ to $70 \mathrm{~mW}$. It can be seen from this result that compared with Ford algorithm, the effectiveness of raising cell-edge power is relatively higher by the proposed algorithm, which also enables to raise the actual SINR values for cell-edge users. From the above comparison, powers of both cell-center user and cell-edge user are raised higher by the proposed algorithm than by Fodor algorithm. Especially for cell-edge users, whose states are always in a low-level SINR, its performance is improved effectively by the proposed method, for the reason is that the global optimized power distribution is established among the whole cell. From the above analysis, it can be found that compared with the comparative analysis of unfair/fair rate allocation in [9], the fairness of the proposed algorithm is more or comparable in each cases for cellular users.

However, for the proposed method both takes Lagrange method and Newton iterative method, which belongs to

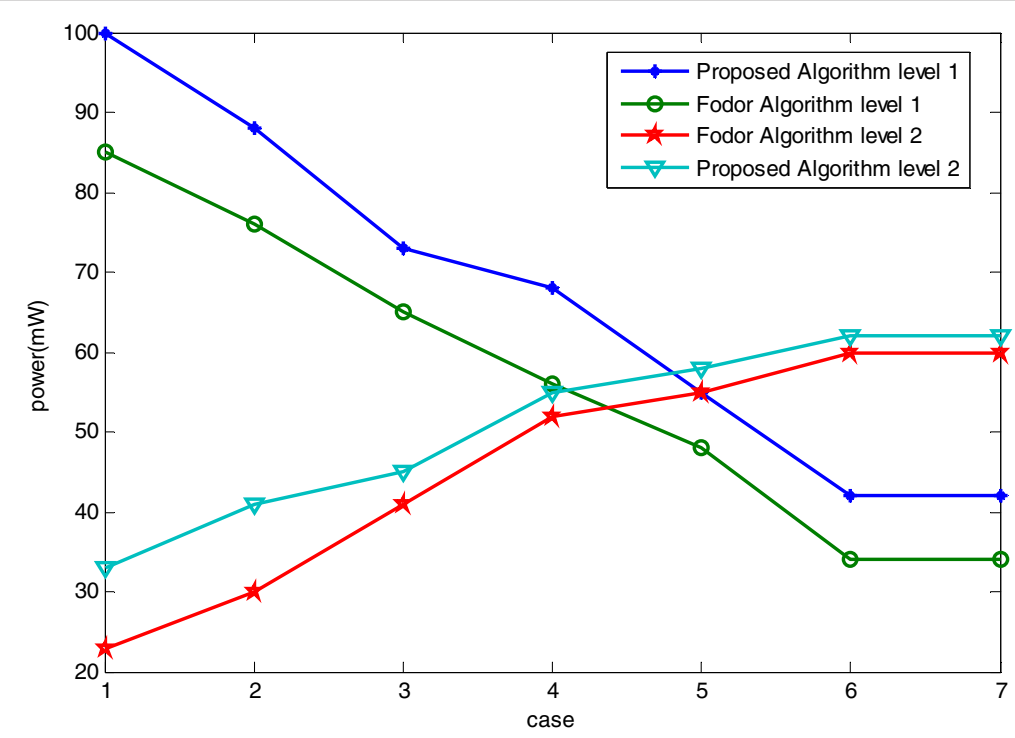

Figure 4 The individual power levels in seven cases. 
the square convergence, and its algorithm complexity is $\mathrm{O}$ $\left(N^{2}\right)$. But the algorithm in [9] only takes Lagrange method, whose convergence speed is faster than the proposed method. Moreover, its complexity is $\mathrm{O}(N)$, which needs less iteration steps than the proposed method. Although the complexity of the proposed method is a little higher than [9], its practical implementation is more adaptive to actual scenario than [9], for the uncertain change of SINR range. Considering the significant increase in complexity, which may be undesirable in cellular networks with mobile users and rapidly changing channel conditions, this analysis result motivates future research to design some measures to decrease the algorithm complexity.

On the other side, in order to verify the performance improvement of both cell-center and cell-edge users brought by the proposed algorithm, we describe the SINR Cumulative Distribution Function (CDF) curve of four cellular users as an example to illustrate it. As shown in Figure 5, it gives the SINR CDF curve for such four users, respectively in cell-center and cell-edge. Among them, user 1 and user 2 are in the cell-center, while user 3 and user 4 are in the cell-edge. Especially, when the CDF value takes $40 \%$, the SINR is around $4 \mathrm{~dB}$. When SINR takes $10 \mathrm{~dB}$, the CDF value is around $70 \%$ to $80 \%$. It can be seen that the CDF distribution of SINR values reach to similar range levels for both cell-center and cell-edge users after taking the proposed algorithm, which shows that the rate close to each other playing the same role with a minimum power, improving users' fairness of experience.

Considering the cell-center users are always with high SINR, while the cell-edge users are always with low SINR, the Figure 6 shows the SINR coverage in cell after using the proposed algorithm, where the $\mathrm{X}$ axis and $\mathrm{Y}$ axis denote the spatial location of user distribution, while the $\mathrm{Z}$ axis denotes the SINR value of current users. About two hundred thousand users are generated by Monte Carlo method and distributed in 19-cell group. It can been seen that the range of SINR is around from $-5 \mathrm{~dB}$ to $15 \mathrm{~dB}$, where the red part denotes stronger SINR area and the blue part denotes lower SINR area. The cell-edge users with better channel conditions could reach to higher range SINR, colour shown as red to yellow. But the performance of cell-edge users with relatively poor channel conditions is improved and the number of such users decrease into a limited range, colour shown as blue, whose coverage is smaller than those red and yellow area. The result illustrates the distribution of users' SINR is optimized. The proposed algorithm reduces the interference power effectively, and the SINR of the users is raised in varying degrees.

\section{Conclusion}

In order to solve the power control problem for multipoint cooperative communication with high-to-low SINR scenario, the cooperative SINR receiving model is established. Considering the power control algorithm problem from its non-convex property, we propose a novel algorithm that is based on geometric programming. On this basis, a series of convex approximations are taken to achieve the global optimization in high-to-low SINR scenario for this algorithm. The numerical results show that the power distribution is improved in both cell-center or cell-edge. Moreover, the CDF of users' SINR is optimized, and the distribution of users' SINR is balanced and more fairness. The SINR of cellular users can be improved in varying degrees whether users in high-SINR area or low-SINR area, which means the inter-cell interference could be effectively mitigated.

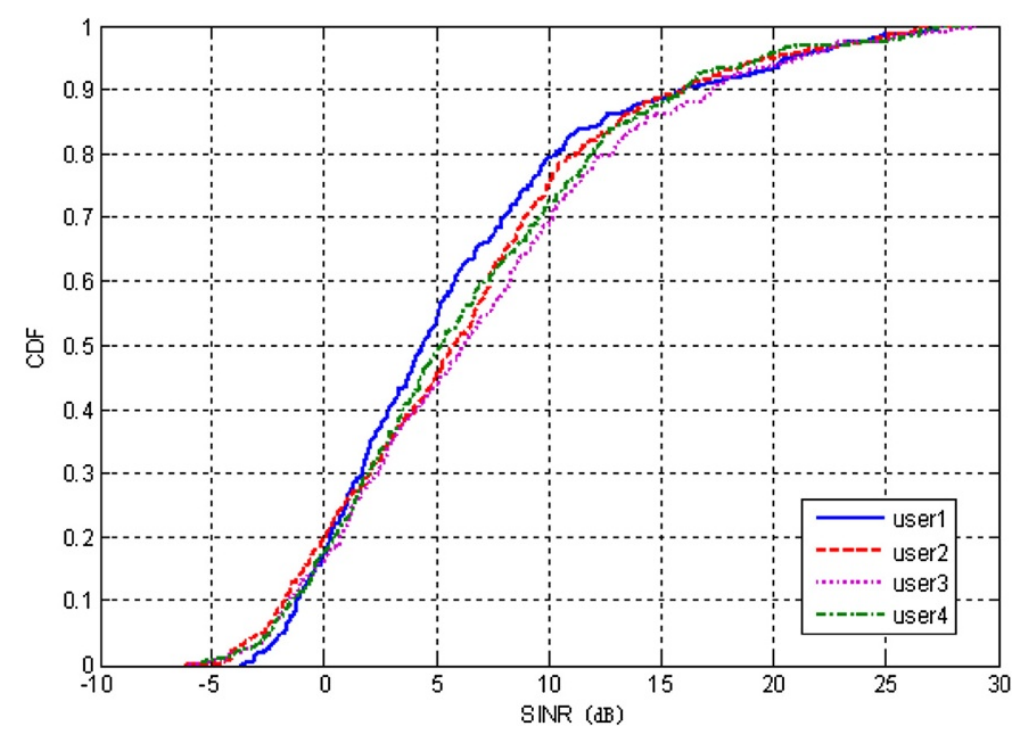

Figure 5 SINR CDF curve for cellular users. 


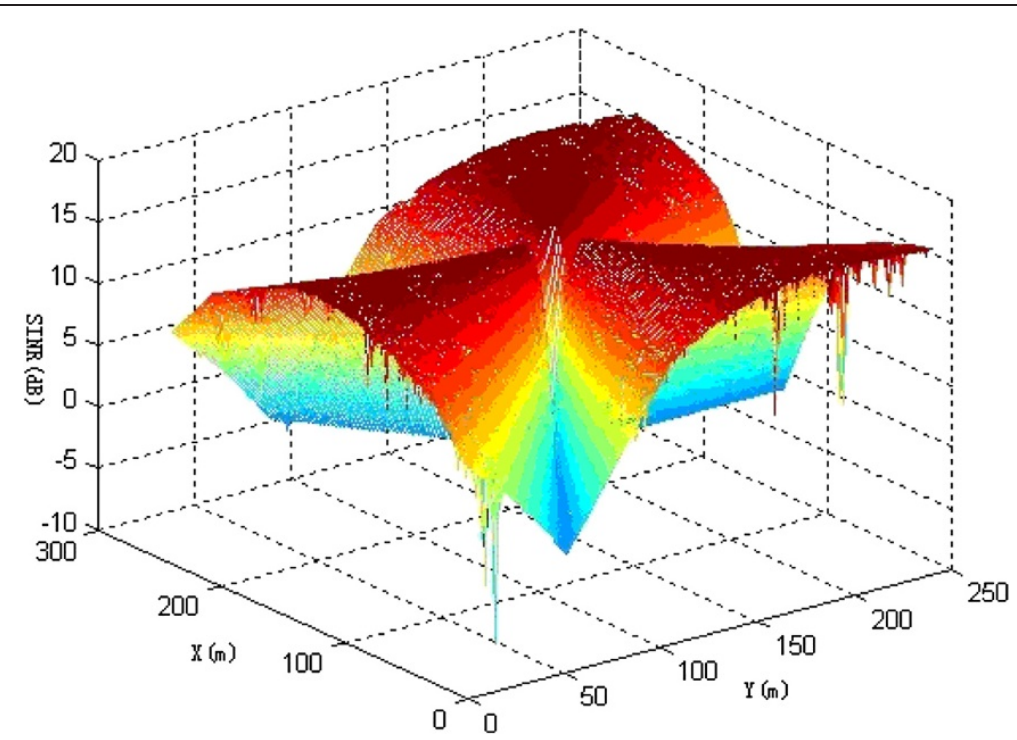

Figure 6 The SINR coverage in cell.

\section{Appendix}

According to (18), this problem is not convex subject to the physical conditions, the solution is given by geometric programming. Considering the no-covexity properties in (18), we try to transit it into convex approximation problem by means of log-sum-exp function in geometric programming. Let $\tilde{P}_{k}=\log P_{k}$, $\tilde{r}_{k}=\log r_{k}$ and $\tilde{n}_{k}=\log n_{k}$, then take an exponential variable transformation $P_{k}=e^{\tilde{P}_{k}}, r_{k}=e^{\tilde{r}_{k}}$ and $n_{k}=e^{\tilde{n}_{k}}$. Moreover, because of $\gamma_{k}=\frac{G_{k} P_{k}}{n_{k}+\sum_{j \neq k} G_{k j} P_{j}}$, where $G_{k k}$ is the link gain matrix, $P_{k}$ is the transmitting power, $n_{k}$ is the Gaussian noise power, $P_{j}$ is the interfered power and $G_{k j}$ is the channel coupling matrix, which is not a concave function of $P_{j}$ [12]. Then, we get the logarithmic form of SINR $\tilde{\gamma}_{k}$ as

$$
\begin{aligned}
\tilde{\gamma}_{k} & =\log \gamma_{k} \\
& =\tilde{P}_{k}+\log \left(G_{k}\right)-\log \left(e^{\tilde{n}_{k}}+\sum_{j \neq k} G_{k j} e^{\tilde{P}_{j}}\right)
\end{aligned}
$$

Considering the Shannon's theory $r_{k}=W \log \left(1+K \gamma_{k}\right)$, which is non-convex in the subject condition. Then we try to get an approximate value. According to the approximate formula for the rate, that is $\tilde{R}_{k}\left(P ; \phi_{k}, \varphi_{k}\right) \triangleq \mathrm{W}\left(\phi_{\mathrm{k}} \log \left(\mathrm{K} \gamma_{\mathrm{k}}\right)+\varphi_{\mathrm{k}}\right)$, so the constrained optimization function is equivalent to (A2)

$$
\begin{gathered}
\underset{P, \gamma}{\operatorname{Maximize}}-\mathrm{P}_{k} \\
\text { subject to } r_{k}^{\text {target }} \leq \tilde{R}_{k}\left(P ; \phi_{k}, \varphi_{k}\right) \\
\sum_{k} e^{\tilde{P}_{k}} \leq P_{\text {tot. }}
\end{gathered}
$$

On this basis, we try to transform the objective function in (A2) into a convex one by means of a series of convex approximations, using a tight iterative inequality between the transmission rate and SINR. By such approximation and the log-transformation, the objective in (18) becomes convex and monotonically increasing. Moreover, the feasibility set is also convex, as shown in (A2). Considering the Lagrange dual function, we get

$$
\begin{aligned}
L\left(\gamma_{k}, \mathrm{P}_{k}\right)= & -\mathrm{P}_{k}+\mu_{k}\left[W\left(\phi_{k} \log \left(K \gamma_{k}\right)+\varphi_{k}\right)-r_{k}^{\text {target }}\right] \\
& +\lambda_{k}\left[P_{\text {tot. }}-\sum_{k} \mathrm{P}_{k}\right]
\end{aligned}
$$

Where $\mu_{k}, \lambda_{k}$ are the Lagrangian multipliers, and the derivation process is as follows

$$
\begin{aligned}
\frac{\partial L\left(\gamma_{k}, \mathrm{P}_{k}\right)}{\partial \mathrm{P}_{k}} & =-1+\frac{\mu_{k} W \phi_{k}}{K \gamma_{k}} \cdot\left(\frac{G_{k}}{n_{k}+\sum_{j \neq k} G_{k j} P_{j}}\right)-\lambda_{k} \\
& =0
\end{aligned}
$$

$$
\begin{aligned}
& \Rightarrow \frac{\mu_{k} W \phi_{k}}{K \gamma_{k}} \cdot\left(\frac{G_{k}}{n_{k}+\sum_{j \neq k} G_{k j} P_{j}}\right)=1+\lambda_{k} \\
& \Rightarrow \frac{\mu_{k} W \phi_{k}}{K\left(1+\lambda_{k}\right)} \cdot\left(\frac{G_{k}}{n_{k}+\sum_{j \neq k} G_{k j} P_{j}}\right)=\gamma_{k} \\
& \Rightarrow \frac{\mu_{k} W \phi_{k}}{K\left(1+\lambda_{k}\right)} \cdot\left(\frac{G_{k}}{n_{k}+\sum_{j \neq k} G_{k j} P_{j}}\right)=\frac{G_{k} P_{k}}{n_{k}+\sum_{j \neq k} G_{k j} P_{j}}
\end{aligned}
$$




$$
\Rightarrow P_{k}=\frac{\phi_{k} \mu_{k} W}{K\left(1+\lambda_{k}\right)}
$$

Similarly, $\frac{\partial L\left(\gamma_{k}, \mathrm{P}_{k}\right)}{\partial \mu_{k}}$ and $\frac{\partial L\left(\gamma_{k}, \mathrm{P}_{k}\right)}{\partial \lambda_{k}}$ can be gotten as following:

$$
\begin{aligned}
& \frac{\partial L\left(\gamma_{k}, \mathrm{P}_{k}\right)}{\partial \mu_{k}}=W\left(\phi_{k} \log \left(K \gamma_{k}\right)+\varphi_{k}\right)-r_{k}^{\text {target }} \\
& \frac{\partial L\left(\gamma_{k}, \mathrm{P}_{k}\right)}{\partial \lambda_{k}}=P_{\text {tot. }}-\sum_{k} \mathrm{P}_{k}
\end{aligned}
$$

On this basis, because of non-linear optimization, we take the Newton iterative method to approach the exact solution gradually. The Newton iterative form is defined as

$$
\begin{aligned}
& \mu_{k}^{(s+1)}=\left[\mu_{k}^{(s)}+\epsilon_{\mu}\left(r_{k}^{\text {target }}-\sum_{n} W\left(\phi_{k} \log \left(K \gamma_{k}^{(s)}\right)+\varphi_{k}\right)\right)\right]_{(\mathrm{A} 11)}^{+} \\
& \lambda_{k}^{(s+1)}=\left[\lambda_{k}^{(s)}+\epsilon_{\lambda}\left(\sum_{n} P_{k}^{n(s)}-P_{\text {tot. }}\right)\right]^{+}
\end{aligned}
$$

Where $[\cdot]^{+}=\max (0,$.$) . By means of (\mathrm{A} 11)$ and (A12), we can get the update form of (A8) according to the Newton iterative method:

$$
P_{k}^{n(s+1)}=\frac{\mu_{k}^{(s)} \phi_{k} W}{K\left(1+\lambda_{k}^{(s)}\right)}
$$

\section{Competing interests}

The authors declare that they have no competing interests.

\section{Acknowledgement}

This research is supported by State Key Laboratory of Networking and Switching Technology Open Project (No. SKLNST-2011-1-02), National Natural Science Foundation Project of China (No. 61101084), the Fundamental Research Funds for the Central Universities, and Specialized Research Fund for the Doctoral Program of Higher Education (No.20110031110028).

\section{Author details}

'State Key Laboratory of Networking and Switching Technology (Beijing University of Posts and Telecommunications), Beijing, China. ${ }^{2}$ Wireless Communication Lab, College of Information Technical Science, Nankai University, 94 Weijin Road, Nankai District, Tianjin, China. ${ }^{3}$ School of Physics and Electronics Engineering, Shanxi University, Taiyuan, Shanxi, China.

Received: 15 December 2011 Accepted: 10 August 2012 Published: 30 August 2012

\section{Reference}

1. W Xin, GB Giannakis, Resource allocation for wireless multiuser OFDM networks. IEEE Transaction on Information Theory 57(7), 4359-4372 (2011)

2. Z Luo, W Yu, An introduction to convex optimization for communications and signal processing. IEEE Journal on Selected Areas in Commun 24(8), 1426-1438 (2006)

3. LP Qian, YJ Zhang, J Huang, Mapel: Achieving global optimality for a nonconvex wireless power control problem. IEEE Trans. on Wireless Comm. 8(3), 1553-1563 (2009)

4. M Chiang, CW Tan, DP Palomar, D O'Neill, D Julian, Power control by geometric programming. IEEE Trans. Wirel. Commun 6(7), 2640-2651 (2007)
5. R Chen, JG Andrews, RW Heath, A Ghosh, Uplink power control in multi-cell spatial multiplexing wireless systems. IEEE Trans. Wirel. Commun 6(7), 2700-2711 (2007)

6. A Ghosh, R Ratasuk, B Mondal, N Mangalvedhe, T Thomas, LTE-advanced: next-generation wireless broadband technology. IEEE Wirel. Commun 17(3), 10-22 (2010)

7. M Sawahashi, Y Kishiyama, A Morimoto, D Nishikawa, M Tanno, Coordinated multipoint transmission/reception techniques for LTE-advanced. IEEE Wirel. Commun 17(3), 26-34 (2010)

8. XH You, DM Wang, B Sheng, XQ Gao, XS Zhao, M Chen, Cooperative distributed antenna systems for mobile communications. IEEE Wirel. Commun 17(3), 35-43 (2010)

9. G Fodor, M Johansson, P Soldati, Near optimum power control under fairness constraints in COMP Systems (Proceedings of the IEEE GLOBECOM, Honolulu, 2009), pp. 1-8

10. J Papandriopoulos, S Dey, J Evans, Optimal and distributed protocols for cross-layer design of physical and transport layers in MANETs. IEEE/ACM Trans. on Net. 16(6), 1392-1405 (2008)

11. H Zhu, S Karachontzitis, D Toumpakaris, Low-complexity resource allocation and its application to distributed antenna systems. IEEE Wireless Communications 17(3), 44-50 (2010)

12. M Chiang, Geometric programming for communication systems. Foundations and Trends in Communications and Information Theory 2(1-2), $1-156(2005)$

13. M Avriel (ed.), Advances in Geometric Programming (Plenum Press, New York, 1980)

14. U Madhow, ML Honig, MMSE interference suppression for direct-sequence spread-spectrum CDMA. IEEE Trans. Commun. 42, 3178-3188 (1994)

15. AF Almutairi, SL Miller, HA Latchman, TF Wong, Power control algorithm for MMSE receiver based CDMA systems. IEEE Commun. Lett 4(11), 346-348 (2000)

16. C Mung, H Prashanth, L Tian, WT Chee, Power control in wireless cellular networks. Found. Trends Netw 2(4), 381-533 (2008)

17. K Phan, S Vorobyov, C Tellambura, T Le-Ngoc, Power control for wireless cellular systems via D.C. programming (Proc. IEEE/SP 14th Workshop on Statistical Signal Processing, Madison, 2007), pp. 507-511

18. M Pischella, J-C Belfiore, Power control in distributed cooperative OFDMA cellular networks. IEEE Trans. Wireless Commun 7(5), 1900-1906 (2008)

19. A Agrawal, JG Andrews, JM Cioffi, T Meng, Iterative power control for imperfect successive interference cancellation. IEEE Trans. Wireless Commun. 4(3), 878-884 (2005)

20. BS-JKL Vandenberghe, S Boyd, J Kim, L Vandenberghe et al., A tutorial on geometric programming. Optimization and Engineering 8, 67-127 (2007)

21. AK Ojha, KK Biswal, Posynomial geometric programming problems with multiple parameters. Journal of Computing 2(1), 84-90 (2010)

22. M Chiang, S Boyd, Geometric programming duals of channel capacity and rate distortion. IEEE Trans. on Inform. Theory 50(2), 245-258 (2004)

23. S Boyd, L Vandenberghe, Convex Optimization. Cambridge, Cambridge Univ (2004). Press

24. J Papandriopoulos, JS Evans, SCALE: A low-complexity distributed protocol for spectrum balancing in multiuser DSL networks. IEEE Trans. on Inform. Theory 55(8), 3711-3724 (2009)

25. JC Ikuno, M Wrulich, M Rupp, System level simulation of LTE networks (IEEE VTC, Taipei, 2010) pp.1 - 5

doi:10.1186/1687-1499-2012-276

Cite this article as: Zhang et al:: Power control for multipoint

cooperative communication with high-to-low sinr scenario. EURASIP

Journal on Wireless Communications and Networking 2012 2012:276. 